\title{
Automatic delineation of drainage basins from contour elevation data using skeleton construction techniques
}

\author{
Giovanni Moretti ${ }^{1}$ and Stefano Orlandini ${ }^{1}$ \\ Received 3 June 2007; revised 7 December 2007; accepted 27 December 2007; published 2 May 2008.
}

[1] New methods for automatic delineation of drainage basins from contour elevation data are presented. As a fundamental preprocessing step, the points defining a set of contour lines are used to compute the Delaunay triangulation, the Voronoi diagram, and other structures known in computational geometry as the crust and the skeleton (or medial axis transform). By exploiting the skeleton extracted from contour lines, a recursive algorithm is then developed to solve critical topographic structures such as ridges, saddles, and peaks in a fully automated and accurate manner. Finally, the algorithm is further extended to deal with the construction of flow nets. Numerical experiments based on high-accuracy contour elevation data of real terrains show that the proposed methods are able to process automatically complex topographic structures and to produce results comparable to those that can be interpreted visually from contour lines. The gain in accuracy over current state-of-the-art solutions is generally found to be significant and to increase as the contour interval increases.

Citation: Moretti, G., and S. Orlandini (2008), Automatic delineation of drainage basins from contour elevation data using skeleton construction techniques, Water Resour. Res., 44, W05403, doi:10.1029/2007WR006309.

\section{Introduction}

[2] The drainage basin determines the area which contributes water and sediments to a given channel cross section and is therefore considered to be the fundamental unit of study of geomorphological and fluvial processes [e.g., Leopold et al., 1964, p. 131]. Using a topographic map, a drainage basin is commonly delineated by searching upslope the lines intersecting contour lines at right angles from the endpoints of the draining cross section [Maxwell, 1870]. Lines drawn at right angles to the contour lines are called slope lines and can be either flow lines (describing the gravity-driven movement of water and sediments) or drainage divides (separating one drainage basin from another). In delineating drainage basins, flow lines are generally drawn so as to connect the draining cross section endpoints to the closest ridges and the drainage divide is drawn along ridges, saddles, and peaks. As an extension of the drainage basin concept, flow nets have been developed to allow a distributed description of gravity-driven transport processes across a drainage basin [e.g., Onstad and Brakensiek, 1968; O'Loughlin, 1986; Moore et al., 1988]. A flow net can be derived by partitioning the drainage basin in a number of elements, each of them being formed by an upper and lower contour segment (for the top and bottom sides of the element) and two flow lines connecting the upper and lower contour lines (for the left and right sides of the element). These flow nets offer, at least in principle, a series of advantages over more efficient and straightforward digital elevation models (e.g., gridded

\footnotetext{
${ }^{1}$ Dipartimento di Ingegneria Meccanica e Civile, Università degli Studi di Modena e Reggio Emilia, Modena, Italy.
}

Copyright 2008 by the American Geophysical Union. 0043-1397/08/2007WR006309\$09.00 (regular network) or triangulated irregular network (TIN) digital elevation models) since they explicitly reproduce the way in which water and sediments flow on the land surface.

[3] Several studies have been carried out to improve the accuracy and the degree of automation in the delineation of drainage basins and construction of flow nets. In the pioneering work by Onstad and Brakensiek [1968], slope lines were specified manually. O'Loughlin [1986] first proposed an automated, robust computation of slope lines, but he did not organize them into a flow net. Moore and Grayson [1991] promoted the potential of flow nets in catchment hydrology, but their automated algorithm for flow net construction was not robust as it required arbitrary adjustment of the boundary in order to operate. Dawes and Short [1994] advanced the treatment of critical points such as peaks and saddles arguing that this is an important task for landscape description. Maunder [1999] strived to make the process of partitioning a catchment into contour-based elements as simple and automated as possible. Menduni and Riboni [2000] and Menduni et al. [2002] emphasized the role of the drainage network in contour-based partitioning of a drainage basin. In fact, all these methods are still semiautomated since they require user-specified procedures to identify and solve accurately some critical topographic structures that may be found in complex terrains. The deficiency of current methods for the automatic delineation of drainage basins and the construction of flow nets, as well as the strategy adopted to remedy this deficiency, are outlined below, after introducing some necessary concepts on the determination of flow lines and drainage divides.

[4] As mentioned above, slope lines (either flow lines or drainage divides) are lines drawn so as to be everywhere at right angles to the contour lines. A curved slope line segment between two adjacent contour lines may therefore 
be drawn in such a way as to be perpendicular to both the upper and lower contour lines. However, this curved slope line segment is not entirely defined, since the morphology of the terrain lying within two adjacent contour lines is unknown, and it must be inferred from the geometry of contour lines. When drawing a curved slope line segment manually, human discernment is used to identify the path that is most likely followed by an imaginary drop of water flowing from the upper contour line to the lower contour line. On the other hand, when slope lines are drawn using a computer program, no inference on the morphology of the terrain lying within adjacent contour lines is normally made. Moreover, a curved slope line segment between two adjacent contour lines is approximated by a (single) straight line segment, which is generally unable to satisfy perpendicularity to both contour lines. As noted by Maxwell [1870] and emphasized by Dawes and Short [1994], in determining ridge lines by searching successively from one contour line to the next, there is a theoretical necessity to consider the minimum distance from points lying on the originating contour line to the upper contour line, by searching upslope the (perpendicular) intersection with the upper contour line, whereas flow lines should be calculated and confluences located by "minimum distance searching downslope." However, as emphasized by Maunder [1999], ridges, saddles, and peaks remains critical topographic structures since large errors may be made when processing these structures even by using Maxwell's [1870] prescriptions, resulting in intersections between adjacent flow lines, flow lines intersecting contour lines at nonright angles, or flow lines that cross the same contour line more than once. These large errors make the automatic solution of complex ridges, saddles, and peaks inaccurate or impossible in most cases, especially when coarse resolution elevation data are used [Dawes and Short, 1994; Maunder, 1999].

[5] The deficiency of current methods that may prevent the accurate and automatic determination of flow lines and drainage divides is ascribed here to the inability of a (single) straight line segment (used to approximate slope lines between adjacent contour lines) to be everywhere at right angles to the contour lines, and, more generally, to the inability of these methods to make inferences on the most plausible morphology of the terrain lying within adjacent contour lines from the set of contour lines considered as a whole. The strategy investigated in this study to remedy this deficiency is based on the application of skeleton construction techniques to contour elevation data. The skeleton (or medial axis transform) of a curve defined by a set of sample points is a geometric structure that can be derived from the Delaunay triangulation and the Voronoi diagram (of this set of sample points), and is fairly well known in computational geometry, computer graphics, and computer vision [Blum, 1967; Kirkpatrick and Radke, 1988; Amenta et al., 1998; Fabbri et al., 2002]. Skeleton construction techniques have recently been applied in terrain modeling to extract accurate TIN from contour elevation data [Gold, 1999; Thibault and Gold, 2000; Gold and Snoeyink, 2001; Dakowicz and Gold, 2003]. The purpose of this investigation is to determine whether the skeleton extracted from contour lines allows for morphological information implicitly present in these contour lines, and normally recognized by human observers, to be explicitly revealed and correctly processed by a computer program, so that critical topographic structures such as ridges, saddles, and peaks can be identified and automatically treated for the delineation of drainage basins and the construction of flow nets.

\section{Methods}

[6] The methods developed in this paper employ fundamental constructs that are known in computational geometry as the Delaunay triangulation, the Voronoi diagram, the crust, and the skeleton (or medial axis transform) [Voronoi, 1907; Delaunay, 1934; Kirkpatrick and Radke, 1988; Amenta et al., 1998]. The definitions of these geometric constructs are given in section 2.1. The skeleton extraction from a set of contour lines is described in section 2.2, whereas the use of this skeleton for the delineation of drainage basins is presented in section 2.3. Section 2.4 shows how methods can be extended to allow the construction of a flow net. Practical aspects are finally reported in section 2.5 .

\subsection{Fundamental Geometric Constructs}

[7] The definitions of Delaunay triangulation, Voronoi diagram, crust, and skeleton are illustrated in Figure 1. A Delaunay triangulation of a set of points in the plane $\left(P_{1}\right.$, $P_{2}, \ldots$ in Figure 1a) is a triangulation (of this set of points) with the property that no point in the set of points falls inside the circumcircle of any triangle (circle which passes through all the three vertices of that triangle) in the triangulation. A Voronoi diagram of a set of points in the plane $\left(P_{1}, P_{2}, \ldots\right.$ in Figure $\left.1 \mathrm{a}\right)$ is a subdivision of the plane into polygonal regions (some of which may be infinite), where each region is that set of points closer to some input point than to any other input point. The Voronoi diagram is the geometric dual of the Delaunay triangulation. The vertices of Voronoi diagrams $\left(V_{1}, V_{2}, \ldots\right.$ in Figure 1a) are the centers of circumcircles associated to Delaunay triangles. The crust and the skeleton of a set of points can be computed on the basis of the related Delaunay triangulation and Voronoi diagram. A Delaunay edge $\left(P_{1} P_{2}\right.$ in Figure 1b) belongs to the crust when a circle exists through its two endpoints (circle in dashed line in Figure 1b) that does not contain either of its associated Voronoi vertices ( $V_{1}$ and $V_{2}$ in Figure 1b). If this condition is not met (the smallest circle through $P_{1}$ and $P_{2}$ drawn in dashed line in Figure 1c, as well as any other circle, does not exclude both the associated Voronoi vertices $V_{1}$ and $V_{2}$ ), then the corresponding Voronoi edge $\left(V_{1} V_{2}\right.$ in Figure 1c) belongs to the skeleton.

[8] As suggested by Gold [1999], crust and skeleton elements can be determined by performing a simple incircle test applied to each Delaunay/Voronoi edge pair. A circle passing through the endpoints of the Delaunay edges $\left(P_{1}\right.$ and $P_{2}$ in Figures $1 \mathrm{~b}$ and $1 \mathrm{c}$ ) and the closest Voronoi vertex ( $V_{1}$ in Figures $1 \mathrm{~b}$ and $1 \mathrm{c}$ ) is drawn (circles in solid line in Figures $1 \mathrm{~b}$ and 1c) and a check is made to assess whether the second Voronoi vertex is inside the circle. A line segment which connects two Delaunay triangle vertices ( $P_{1}$ and $P_{2}$ in Figure $1 \mathrm{~b}$ ) belongs to the crust if by drawing a circle (circle in solid line in Figure 1b) through them and a corresponding Voronoi vertex ( $V_{1}$ in Figures $\left.1 \mathrm{~b}\right)$, the circle does not include the second Voronoi vertex ( $V_{2}$ in Figure $\left.1 b\right)$. A line segment which connects two Voronoi vertices $\left(V_{1}\right.$ and $V_{2}$ in Figure 1c) belongs to the skeleton if the circle 


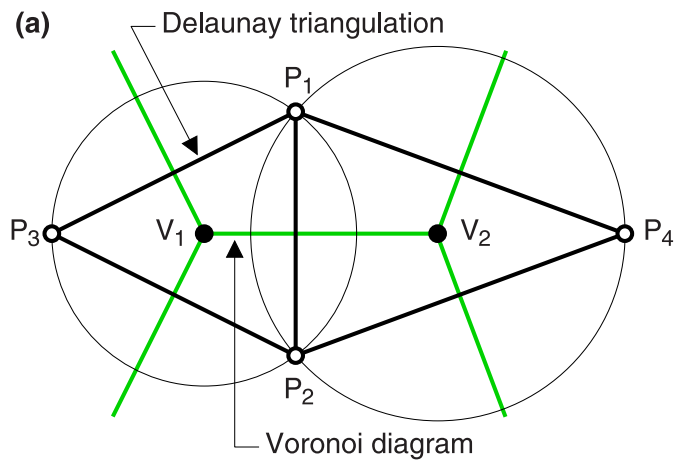

(b)
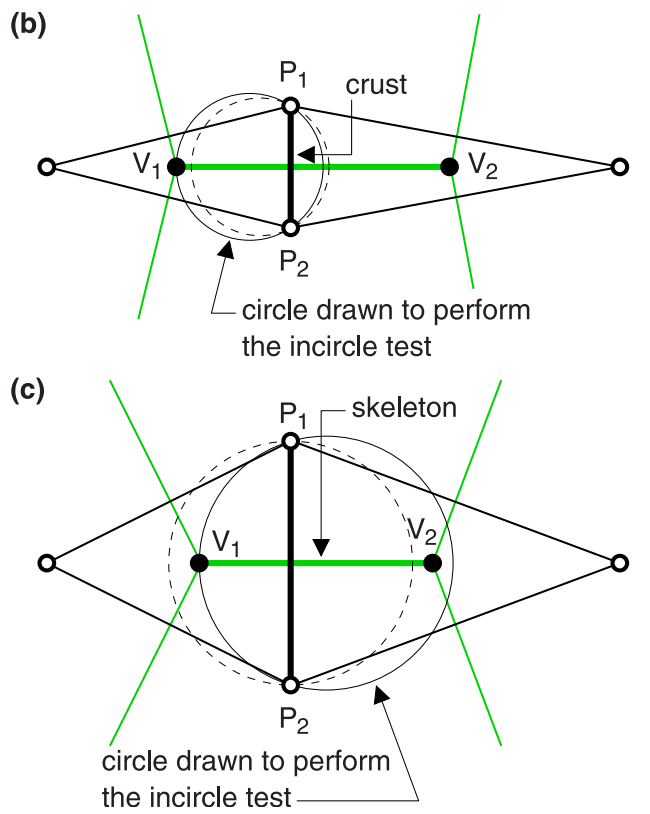

Figure 1. Definition of the fundamental geometric constructs used by the proposed method: (a) Delaunay triangulation and Voronoi diagram, (b) crust, and (c) skeleton.

(circle in solid line in Figure 1c) through one of them $\left(V_{1}\right.$ in Figure 1c) and the two corresponding Delaunay triangle vertices $\left(P_{1}\right.$ and $P_{2}$ in Figure $\left.1 \mathrm{c}\right)$ also include the second one $\left(V_{2}\right.$ in Figure $\left.1 \mathrm{c}\right)$.

[9] Amenta et al. [1998] showed in their seminal study that the crust and the skeleton extracted from a set of sample points lying on a curve can be used to reconstruct an approximation of this curve. The procedure suggested by Amenta et al. [1998] is illustrated in Figures $2 \mathrm{a}-2 \mathrm{c}$ in the simple case of a set of sample points lying on the border of a rectangle. This set of sample points (Figure $2 \mathrm{a}$ ) is used to compute the Delaunay triangulation and the Voronoi diagram (Figure 2b), which allow for the extraction of a crust that is found to reconstruct the border of the rectangle (Figure 2c). Clearly, no algorithm for the computation of the crust can reconstruct any curve from any set of sample points and some condition on the quality of the set of sample points is needed. Amenta et al. [1998] found that the reconstruction of a curve is guaranteed if the distance from any point of the curve to the nearest sample point is less than or equal to 0.252 times the distance between this point (of the curve) to the nearest point of the computed skeleton. The concepts illustrated in this section can be applied to closed curves such as that considered in Figures $2 a-2 c$, or to a set of open curves such as that shown in Figures $2 \mathrm{~d}-2 \mathrm{f}$.

\subsection{Skeleton Extraction From Contour Lines}

[10] As first noted by Gold [1999], the theory developed by Amenta et al. [1998] can be applied, although from a different perspective, to a set of contour lines exhibiting the morphology of a terrain. While Amenta et al. [1998] were mostly interested in the crust as a means for reconstructing a curve from a given set of sample points, in terrain analysis the curves, namely the contour lines, are given (i.e., they need not to be reconstructed) and the attention is rather focused on the skeleton of the contour lines as a possible means for reconstructing the most plausible morphology of the terrain lying within adjacent contour lines or within a closed contour line. The simple cases shown in Figure 2 may be used as examples to explain the rationale of this strategy. Assuming that the rectangle's border is a contour line defined by a set of points (Figure 2a), it can be observed that the crust reconstructs the contour line and the skeleton reveals a plausible morphology of the terrain within this contour line (Figure 2c). If the contour line contains a peak (and not a depression), then the skeleton provides the ridge lines for the terrain lying within this contour line, revealing a plausible "hipped roof geometry" of the peak. The skeleton in the central part of the rectangle is generated from opposite (parallel) rectangle sides and represents in this case the main ridge of the roof, whereas the skeleton in the regions near the corners of the rectangle is generated from contiguous (markedly nonparallel) rectangle sides and represents in this case the secondary ridges running along the hips of the roof. It is noted here that the skeleton may be obtained by connecting Voronoi vertices lying outside the associated Delaunay triangles (see, for instance, the Delaunay triangle $P_{1} P_{2} P_{3}$ and the related Voronoi vertex $V$ in Figure 2b).

[11] The open curves defined by a set of points as shown in Figure $2 \mathrm{~d}$ are assumed here to represent two contour lines representing different elevations, with the higher elevation represented by the (straight) top contour line. As shown in Figures $2 \mathrm{e}$ and $2 \mathrm{f}$, the skeleton resulting from Delaunay triangles with vertices distributed over both the two contour lines provides a sort of intermediate contour line, whereas the skeleton resulting from Delaunay triangles with all vertices on the same contour line provides a ridge line. The simple cases shown in Figure 2 can be used to illustrate a classification of skeleton lines computed from points defining contour lines that is crucial for the development of the methods presented in this study. A skeleton line generated from a set of points lying on contour lines representing different elevations is a skeleton stem (line labeled "skeleton stem" in Figure 2f), whereas a skeleton line generated from a set of points lying on the same contour line or on two contour lines representing the same elevation is a skeleton branch (lines labeled "skeleton branch" in Figures 2c and 2f). It is noted here that Delaunay triangles with all three vertices lying on the same contour line, which are problematic cases when standard (TINbased) methods for the description of terrain morphology are used, do not constitute a critical point when skeleton construction techniques are used, and are rather crucial to determine skeleton branches revealing important morphological features of the terrain [Gold, 1999]. The theory 
(a)

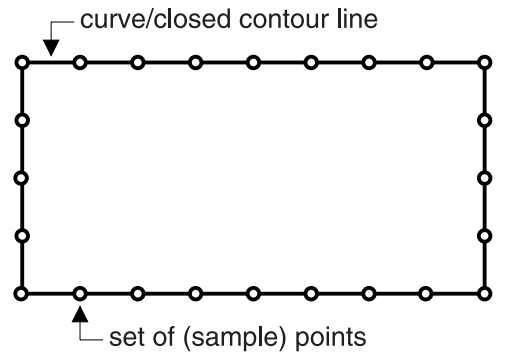

(b) Delaunay triangulation

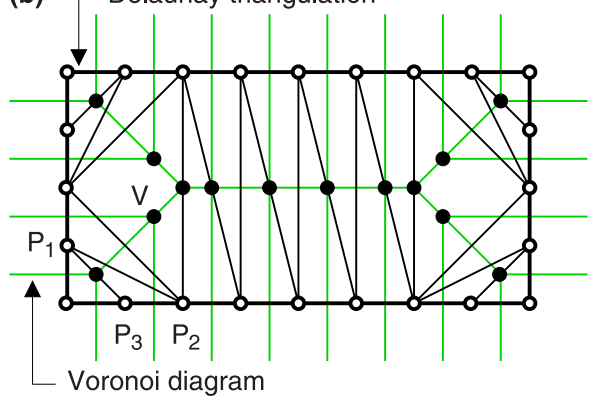

(c)

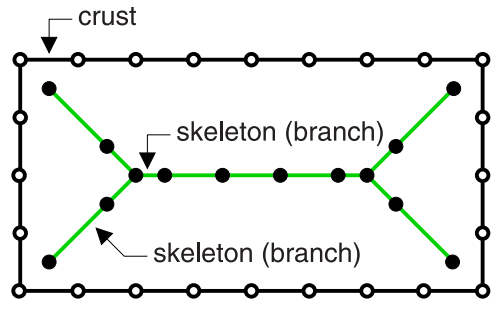

(d)

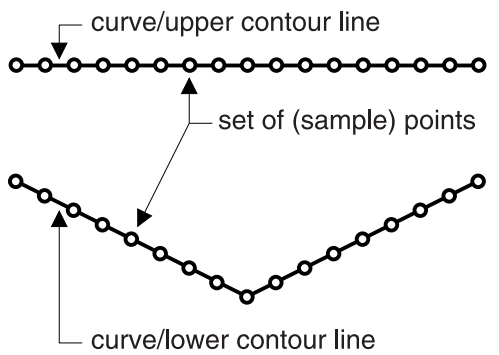

(e)

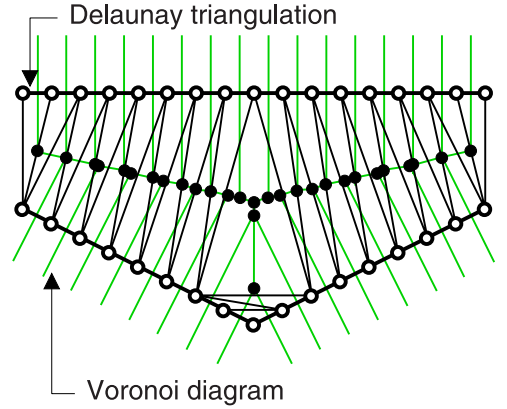

(f)

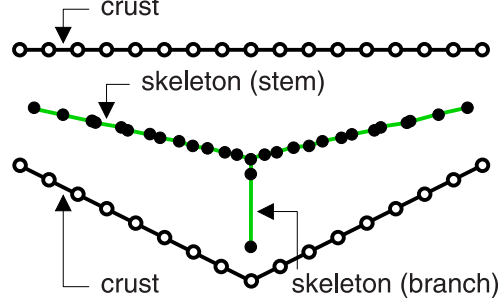

Figure 2. Computation of the crust and the skeleton from a set of (sample) points lying ( $a, b$, and $c)$ on the border of a rectangle and (d, e, and $\mathrm{f}$ ) on two adjacent open curves: set of sample points (Figures $2 \mathrm{a}$ and 2d), Delaunay triangulation and Voronoi diagram (Figures $2 \mathrm{~b}$ and $2 \mathrm{e}$ ), and crust and skeleton (Figures 2c and 2f).

developed by Amenta et al. [1998] ensures that, if contour lines are defined by a sufficiently dense set of points, the associated crust reconstructs these contour lines and the associated skeleton reveals a plausible description of the morphology of the terrain lying within the contour lines. The issues about the density of sample points introduced in section 2.1 are not critical for the problem addressed in this study since contour lines are given and a sufficiently dense set of points lying on these contour lines may always be generated, if necessary, to meet the well-defined requirements provided by Amenta et al. [1998].

[12] Further details on the skeleton extracted from a real set of contour lines can be provided by referring to the cases shown in Figure 3. As shown in Figure 3a, between adjacent contour lines representing different elevations a skeleton stem is always generated and provides a sort of intermediate contour line (lines labeled "skeleton stem"). Where contour lines are markedly nonstraight, the skeleton is branched and skeleton branches (lines labeled "skeleton branch") generally form a tree structure that can be ordered in such a way that the first-order skeleton branches are those which connect the skeleton stem to the closest contour line (skeleton branch labeled " 1 "), the second-order skeleton branches are those which connect first-order skeleton branches to the same contour line (skeleton branches labeled " 2 "), and similarly for higher-order skeleton branches. It is specified here that in this study the endpoint of each skeleton branch is connected to the closest contour line in order to provide a continuous system formed by the contour lines and the related skeleton structure. The skeleton branches reveal plausible morphologic features such as minor ridges or valleys as described in the following paragraphs.

[13] A ridge connecting two contour lines representing higher elevations indicates a saddle (line segments $S_{L}^{(n)} S_{R}^{(n)}$ in Figures $3 b$ and $3 c$ ). As shown in Figures $3 b$ and $3 c$, a firstorder skeleton branch that has both the endpoints belonging to skeleton stems (and neither of them belonging to the contour line) is used to identify and characterize a saddle. Two types of saddle can be found: type 1 saddles are those identified by a skeleton branch with both the endpoints lying on the same skeleton stem (see the small inset below the label "type 1 saddle" in Figure 3b), whereas type 2 saddles are those identified by a skeleton branch with endpoints lying on different skeleton stems (see the small inset below the label "type 2 saddle" in Figure 3c). In practice, the two different types of saddles are distinguished by considering the Delaunay triangles associated to the endpoints of the skeleton branch that identifies and characterizes the saddle. A type 1 or type 2 saddle is identified depending on whether two (out of three) vertices of these triangles lie on the upper (e.g., triangle $P_{1} P_{2} P_{3}$ in Figure $3 \mathrm{~b}$ ) or lower (e.g., triangle $P_{1} P_{2} P_{3}$ in Figure $3 \mathrm{c}$ ) contour lines, respectively. It is noted here that the midpoint of the saddle branch used to identify and characterize the type 1 and 

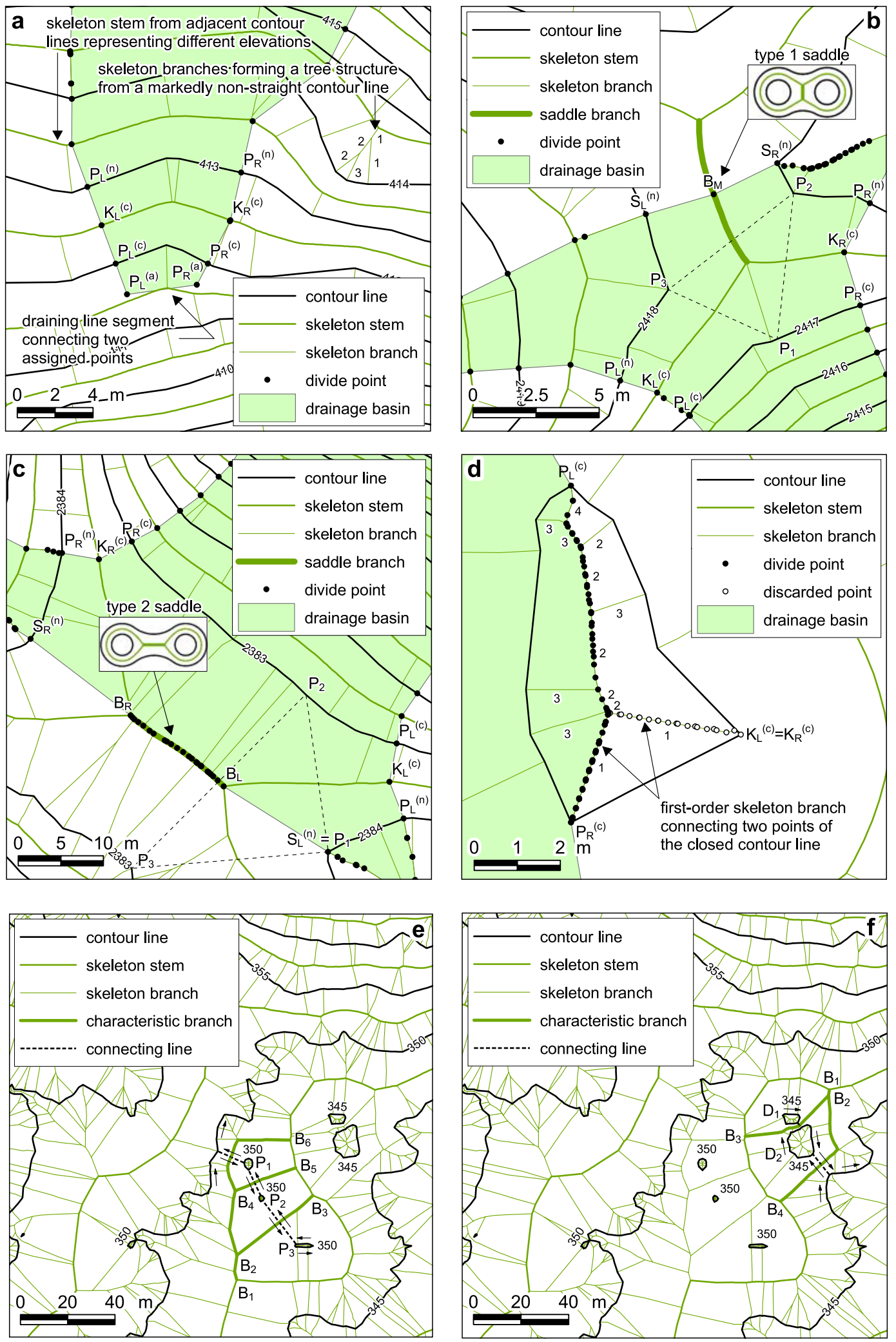

Figure 3. Essential features of the skeleton extracted from contour lines and of the drainage basin delineation algorithm developed by exploiting this skeleton: (a) draining line segment connecting two assigned points and upslope searching algorithm, (b) type 1 saddle identification and solution, (c) type 2 saddle identification and solution, (d) peak identification and closure of the drainage basin, (e) group of peaks lying between adjacent contour lines, and (f) group of depressions lying between adjacent contour lines. The flow net for approximately the same area as that shown in Figures $3 \mathrm{e}$ and $3 \mathrm{f}$ is given in Figure $6 \mathrm{~b}$. 
type 2 saddles determines the saddle point. In type 1 saddles, the characteristic saddle branch curves down and is used to determine a transverse saddle line $S_{L}^{(n)} S_{R}^{(n)}$ which curves up. In type 2 saddles, the characteristic saddle branch curves up and belongs to the saddle line $S_{L}^{(n)} S_{R}^{(n)}$ which clearly curves up as well.

[14] A region lying within a closed contour line (with no enclosed contour lines) indicates a peak or a depression (Figure 3d). The closed contour line describes a peak or a depression depending on whether the surrounding contour lines represent lower or higher elevations, respectively. As shown in Figure 3d, the skeleton contained within the closed contour line is used to identify and characterize the peak or depression. The skeleton stem is not present and the skeleton branches are ordered using a system similar to that introduced above. The first-order skeleton branch (skeleton branch labeled " 1 ") connects two points of the closed contour line $\left(P_{R}^{(c)}\right.$ and $\left.K_{R}^{(c)}\right)$, second-order skeleton branches (skeleton branches labeled " 2 ") are those which connect the first-order skeleton branch to the closed contour line, thirdorder skeleton branches (skeleton branches labeled " 3 ") are those which connect the second-order skeleton branches to the closed contour line, and similarly for higher-order skeleton branches. The skeleton branches lying within a closed contour line describing a peak or a depression reveal a system of ridge lines or flow lines, respectively.

[15] When a complex terrain is represented by a set of contour lines having a contour interval that is too large to yield a detailed description of the terrain morphology, topographic structures composed of groups of peaks and/ or depressions lying between adjacent contour lines may be observed (Figures 3e and 3f). As shown in Figures 3e and $3 \mathrm{f}$, in the region lying within the adjacent open contour lines representing the elevations $345 \mathrm{~m}$ asl (above sea level) and $350 \mathrm{~m}$ asl, the closed contour lines representing the elevation $350 \mathrm{~m}$ asl (those labeled $P_{1}, P_{2}$, and $P_{3}$ in Figure $3 \mathrm{e}$ ) describe a group of peaks since they represent an elevation that is equal to the elevation of the upper contour line (350 $\mathrm{m}$ asl), whereas the closed contour lines representing the elevation $345 \mathrm{~m}$ asl (those labeled $D_{1}$ and $D_{2}$ in Figure 3f) describe a group of depressions since they represent and elevation that is equal to the elevation of the lower contour line $(345 \mathrm{~m}$ asl). The cases under consideration can be identified and characterized using the skeleton of contour lines. However, the skeleton elements need to be analyzed and the closed contour lines representing peaks or depressions need to be connected to the upper or the lower contour line, respectively, in order to provide a well-defined, plausible characterization of the morphology of the terrain lying within the adjacent contour lines. Although the group of peaks and the group of depressions are solved using the same methods, these two cases are dealt with separately in the following paragraphs.

[16] As shown in Figure 3e, the topographic structure composed of the open contour line representing the elevation $350 \mathrm{~m}$ asl and of the closed contour lines representing the elevation $350 \mathrm{~m}$ asl (peaks $P_{1}, P_{2}$, and $P_{3}$ ) can be recognized from the skeleton branches labeled "characteristic branch" ( $B_{1} B_{2}, B_{2} B_{3}, B_{2} B_{4}, B_{4} B_{5}$, and $\left.B_{4} B_{6}\right)$. These characteristic branches are derived from Delaunay edges connecting points with the same elevation (as occurs for all skeleton branches) and lying on different contour lines (as occurs for the saddle branches shown in Figures $3 b$ and $3 c$ ), but are characteristic since at least one of their endpoints lies on a skeleton branch (and not both their endpoints lie on one or two skeleton stems as occurs for the type 1 and type 2 saddle branches shown in Figures $3 b$ and $3 c$, respectively). Each closed contour line describing a peak may be connected to the open contour line on the basis of two simple rules: (1) the connection may be direct or achieved through the other closed contour lines describing the peaks, and (2) among the possible connections, that displaying the minimum distance is chosen (lines labeled "connecting line"). In the example shown in Figure $3 \mathrm{e}$, the connecting lines are those intersecting the characteristic branches $B_{2} B_{3}$, $B_{4} B_{5}$, and $B_{4} B_{6}$. The morphology of the group of peaks is well defined by the characteristic branches and the obtained connecting lines. It is noted that (1) the intersection between a connecting line and the related characteristic branch defines a saddle point, (2) a connecting line curves up and represents a saddle line connecting two higher elevations (350 m asl), and (3) a characteristic branch intersected by a connecting line curves down and defines two plausible flow paths originating at the saddle point and extending along the two opposite sides of the saddle. A characteristic branch that is not intersected by a connecting line $\left(B_{1} B_{2}\right.$ and $\left.B_{2} B_{4}\right)$ does not identify a saddle but rather a simple flow path.

[17] As shown in Figure 3f, the topographic structure composed of the open contour line representing the elevation $345 \mathrm{~m}$ asl and of the closed contour lines representing the elevation $345 \mathrm{~m}$ asl and describing the depressions $D_{1}$ and $D_{2}$ can be recognized from the skeleton branches labeled "characteristic branch" $\left(B_{1} B_{2}, B_{2} B_{3}\right.$, and $\left.B_{2} B_{4}\right)$. These characteristic branches are determined as described above when dealing with the case of the group of peaks. In the example shown in Figure $3 f$, the connecting lines are those intersecting the characteristic branches $B_{2} B_{3}$ and $B_{2} B_{4}$, and are determined as described above when dealing with the case of the group of peaks. In this case, however, it is noted that (1) the intersection between a connecting line and the related characteristic branch defines a saddle point, (2) a characteristic branch intersected by a connecting line curves up and represents a saddle line connecting two higher elevations, and (3) a connecting line curves down and defines two plausible flow paths originating at the saddle point and extending along the two opposite sides of the saddle. A characteristic branch that is not intersected by a connecting line $\left(B_{1} B_{2}\right)$ does not identify a saddle but rather a simple ridge line.

\subsection{Drainage Basin Delineation}

[18] A recursive algorithm for the delineation of drainage basins that exploits the skeleton computed from a set of contour lines is presented in this section. The essential features of this algorithm are illustrated by considering the topographic structures shown in Figure 3. The real case applications shown in Figures 4 and 5 are also used to provide some illustrative examples of the behavior of the proposed method with respect to methods currently in use. These real case applications will be described in more detail in section 3 . The algorithm constructs the drainage divide from two assigned points $\left(P_{L}^{(a)}, P_{R}^{(a)}\right.$ in Figure 3a) for each pair of adjacent contour lines working from the lowest to the highest contour line. Subscripts " $L$ " and " $R$ " are used in 


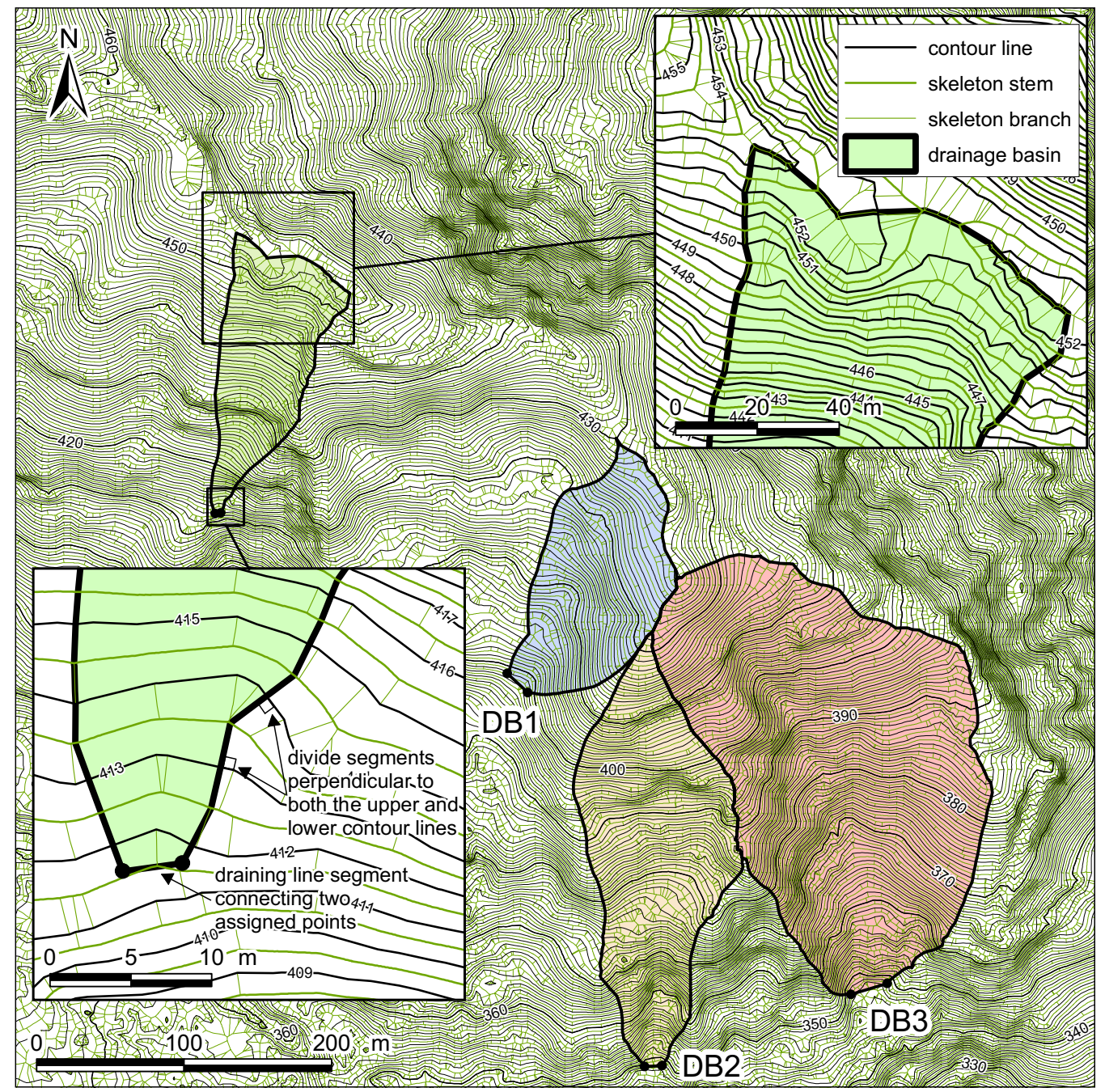

Figure 4. Delineation of drainage basins in the Ca' Lita area $\left(44^{\circ} 27^{\prime} 31^{\prime \prime} \mathrm{N}, 10^{\circ} 38^{\prime} 38^{\prime \prime} \mathrm{E}\right)$ using the proposed method. The essential features of the delineation method are shown in the insets. The three drainage basins labeled DB1, DB2, and DB3 are used to evaluate the method numerically as described in section 3.2. The contour interval is $1 \mathrm{~m}$. The datum is mean sea level.

this context to denote the "left" and "right" relative locations of two points for an observer located within the drainage basin and looking upstream. Superscripts " $(a)$," " $(c)$, , and " $(n)$ " are used to denote the "assigned," "current," and "next" points, respectively, in the step-bystep determination of the drainage divide. A drainage divide element between two adjacent contour lines (e.g., element $P_{R}^{(c)} K_{R}^{(c)} P_{R}^{(n)}$ in Figure 3a) is normally formed by a couple of contiguous straight line segments (e.g., $P_{R}^{(c)} K_{R}^{(c)}$ and $K_{R}^{(c)} P_{R}^{(n)}$ in Figure 3a) joined at a point lying on the skeleton stem (e.g., $K_{R}^{(c)}$ in Figure $3 \mathrm{a}$ ), and oriented in such a way as to intersect both the lower and upper contour lines at right angles (e.g., at points $P_{R}^{(c)}$ and $P_{R}^{(n)}$, respectively, in Figure 3a). This capability of the proposed method, highlighted in the bottom inset of Figure 4, allows to remedy a major deficiency of current methods mentioned in section 1 and highlighted in the bottom inset of Figure 5.

[19] The assigned points $\left(P_{L}^{(a)}, P_{R}^{(a)}\right.$ in Figure 3a) can be chosen arbitrarily along a selected contour line or in the region lying within two adjacent contour lines. The divide segments originating at the assigned points $\left(P_{L}^{(a)}, P_{R}^{(a)}\right)$ are identified by distinguishing the cases in which these assigned points lie immediately downslope from a contour or a skeleton stem line. In the first case (shown in Figure 3a), from the assigned points the lines perpendicular to the upper contour line are computed and the points of intersection $\left(P_{L}^{(c)}, P_{R}^{(c)}\right)$ determined. In the second case (not shown in Figure $3 \mathrm{a}$ ), the lines perpendicular to the lower contour line and passing through the assigned points are computed and the points of intersection between these lines and the upper skeleton stem line $\left(K_{L}^{(c)}, K_{R}^{(c)}\right)$ are determined. From these skeleton points the lines perpendicular to the upper contour line are searched upslope and the points of intersection $\left(P_{L}^{(c)}\right.$, $\left.P_{R}^{(c)}\right)$ determined. Having found the current points $\left(P_{L}^{(c)}, P_{R}^{(c)}\right)$ on the first contour line lying upward from the assigned points $\left(P_{L}^{(a)}, P_{R}^{(a)}\right)$, the recursive algorithm can initiate.

[20] The divide elements connecting two adjacent contour lines are determined in two phases. In the first phase, the lines perpendicular to the lower contour line from the current points $\left(P_{L}^{(c)}, P_{R}^{(c)}\right.$ in Figure $\left.3 \mathrm{a}\right)$ are computed and the points of intersection with the skeleton stem $\left(K_{L}^{(c)}, K_{R}^{(c)}\right.$ in Figure $3 a)$ determined. In some specific cases, where this 


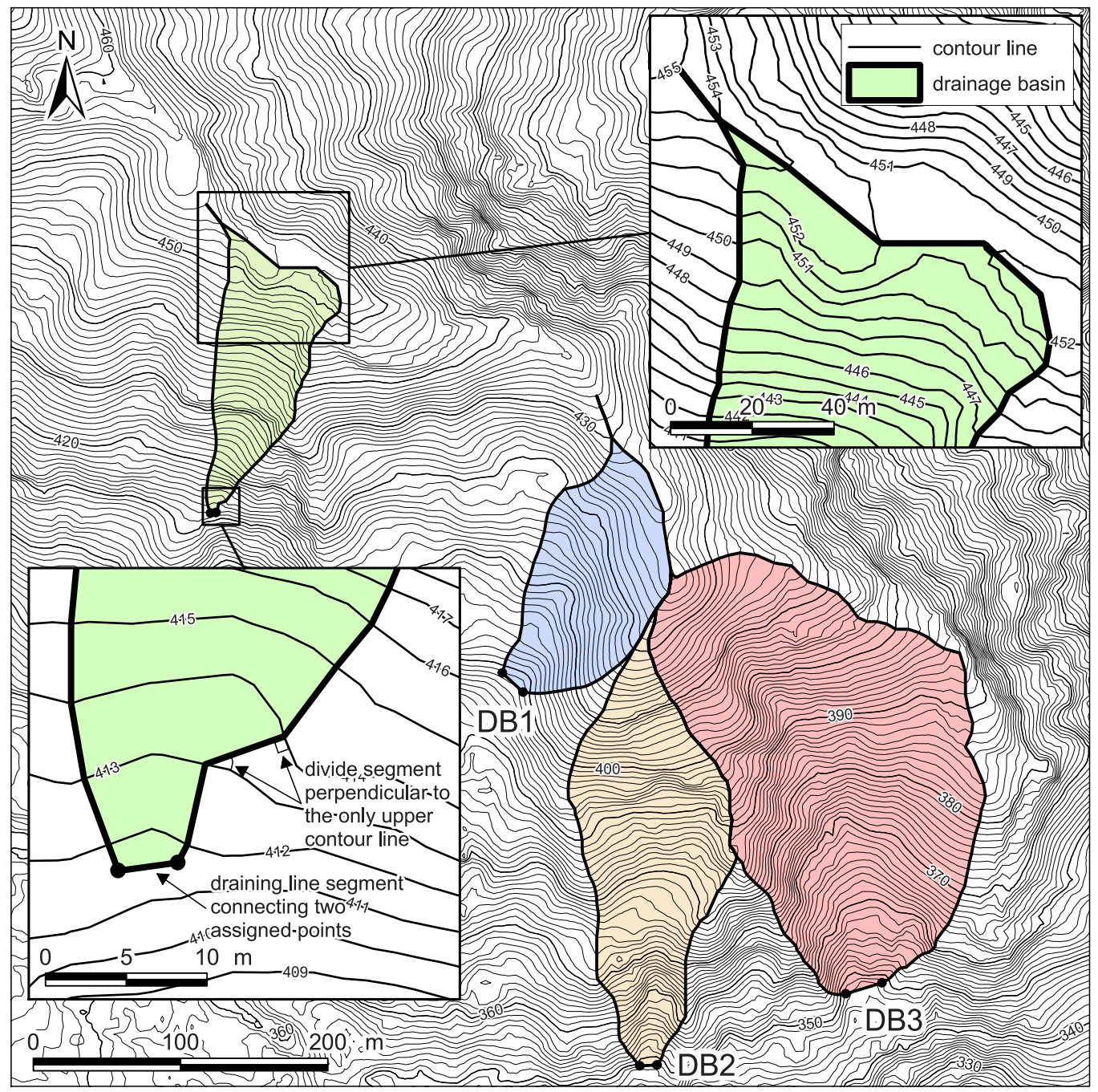

Figure 5. Delineation of drainage basins in the Ca' Lita area $\left(44^{\circ} 27^{\prime} 31^{\prime \prime} \mathrm{N}, 10^{\circ} 38^{\prime} 38^{\prime \prime} \mathrm{E}\right)$ using the current state-of-the-art method. The essential features of the delineation method are shown in the insets. The three drainage basins labeled DB1, DB2, and DB3 are used to evaluate the method numerically as described in section 3.2. The contour interval is $1 \mathrm{~m}$. The datum is mean sea level.

line meets a skeleton branch (before the intersection with the skeleton stem), the divide is forced to follow this skeleton branch, and eventually other lower-order skeleton branches until the skeleton stem is reached. In the second phase, the minimum distances from the points lying on the skeleton stem $\left(K_{L}^{(c)}, K_{R}^{(c)}\right.$ in Figure $\left.3 \mathrm{a}\right)$ to the upper contour line is computed and the points of intersection $\left(P_{L}^{(n)}, P_{R}^{(n)}\right.$ in Figure 3a) are determined. This "minimum distance searching upslope" criterion normally allows that the drainage divide intersects the upper contour line at right angle. At some singular points the upper contour line may not be smooth (e.g., it may display a cusps). The algorithm (automatically) identify these singular points and use them to delineate the drainage basin if they satisfy the criterion of minimum distance (although with no intersection at right angle). When the specific cases mentioned above are not met, the proposed method ensures that upslope and downslope searching will provide the same results.

[21] As mentioned above, the delineation of the drainage basin divide is carried out step by step, using at each step the current points $\left(P_{L}^{(c)}, P_{R}^{(c)}\right)$ to determine the next points
$\left(P_{L}^{(n)}, P_{R}^{(n)}\right)$. The procedure continues as long as the points $K_{L}^{(c)}, K_{R}^{(c)}$ lying on the skeleton stem upward from the current points $\left(P_{L}^{(c)}, P_{R}^{(c)}\right)$ do not coincide or, in other terms, until a peak is found. If the two next points are found to lie on different contour lines, a saddle identification procedure is applied. If a type 1 saddle is met (Figure $3 \mathrm{~b}$ ), the midpoint $\left(B_{M}\right)$ of the associated skeleton branch is determined and the two closest next points $\left(S_{L}^{(n)}, S_{R}^{(n)}\right)$ among those of the upper contour lines are searched. If a type 2 saddle is met (Figure 3c), the endpoints $\left(B_{L}, B_{R}\right)$ of the associated skeleton branch are considered and the two closest next points $\left(S_{L}^{(n)}, S_{R}^{(n)}\right)$ among those of the upper contour lines are searched. In both type 1 and type 2 saddle cases, two pairs of points are obtained. One pair, conventionally denoted as the left one $\left(P_{L}^{(n)}, S_{L}^{(n)}\right.$ in Figures $3 \mathrm{~b}$ and $\left.3 \mathrm{c}\right)$ are considered as the input for a new call of the recursive procedure. This call terminates when a peak is found. As shown in Figure 3d, a peak is normally described by a closed contour line with no enclosed contour lines and by an ordered set of skeleton branches within the closed contour line. From each of the current points $\left(P_{L}^{(c)}, P_{R}^{(c)}\right)$, the set of skeleton branches is 

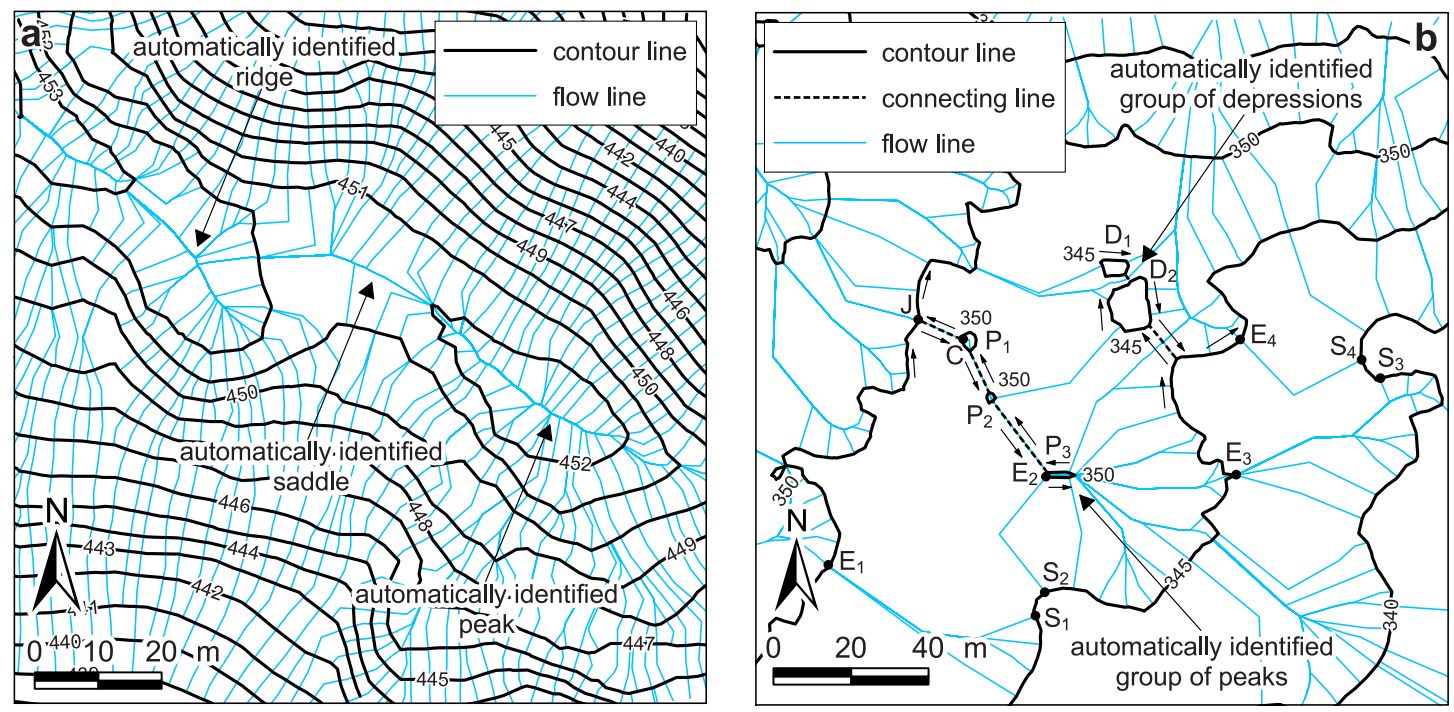

Figure 6. Automatically constructed flow nets for two portions of the Ca' Lita area showing (a) the solution of critical topographic structures such as ridges, saddles, and peaks $\left(44^{\circ} 27^{\prime} 56^{\prime \prime} \mathrm{N}, 10^{\circ} 38^{\prime} 22^{\prime \prime} \mathrm{E}\right)$ and (b) the solution of a group of peaks and/or depressions $\left(44^{\circ} 27^{\prime} 18^{\prime \prime} \mathrm{N}, 10^{\circ} 38^{\prime} 16^{\prime \prime} \mathrm{E}\right)$. The contour interval is $1 \mathrm{~m}$ in Figure $6 \mathrm{a}$ and $5 \mathrm{~m}$ in Figure $6 \mathrm{~b}$. The datum is mean sea level.

traveled in the direction of descending skeleton branch order (filled and empty circles in Figure 3d) until the first endpoint of the first-order skeleton branch is reached (point $K_{L}^{(c)}=K_{R}^{(c)}$ in Figure $3 \mathrm{~d}$ ). The points belonging to both the left and right parts of the basin divide within the closed contour line (empty circles in Figure $3 \mathrm{~d}$ ) are then discarded. It is specified here that the overlap of point $P_{R}^{(c)}$ with the end of the first-order skeleton branch, as well as the overlap of point $P_{L}^{(c)}$ with the end of the fourth-order skeleton branch in Figures $3 \mathrm{~d}$, are coincidental. In the general case, these points may belong to any point of the closed contour line. From these points the lines perpendicular to the contour line are computed and the points of intersection with the skeleton branches lying within the contour line are determined. Once the drainage divide through the left points $\left(P_{L}^{(n)}, S_{L}^{(n)}\right)$ is solved, the algorithm for the delineation of the whole drainage basin continues by considering the right pair of points $\left(S_{R}^{(n)}, P_{R}^{(n)}\right.$ in Figures $3 \mathrm{~b}$ and $\left.3 \mathrm{c}\right)$. When a new saddle is found, a new saddle solution routine is recursively called. An illustrative example of the accuracy with which critical topographic structures such as ridges, saddles, and peaks are solved by the proposed method is given in the top inset of Figure 4, whereas the top inset of Figure 5 shows a less accurate solution of the same problem obtained by using the current state-of-the-art methods.

[22] The following pseudocode (starting with the current points of the first contour line lying upslope from the assigned points as described in the first paragraph of this section) gives the logic of the algorithm:

Procedure $\operatorname{DIVIDE}\left(P_{L}^{(c)}, P_{R}^{(c)}\right)$

Require: Contour lines, skeleton, points $\left(P_{L}^{(c)}, P_{R}^{(c)}\right)$

Ensure: Drainage divide through the two points $\left(P_{L}^{(c)}, P_{R}^{(c)}\right)$

$K_{L}^{(c)}=$ point of the skeleton searched upslope $P_{L}^{(c)}$

$K_{R}^{(c)}=$ point of the skeleton searched upslope $P_{R}^{(c)}$ while $K_{L}^{(c)} \neq K_{R}^{(c)}$ do

\{Until a PEAK is found:\}

$P_{L}^{(n)}=$ point of the contour line searched upslope $K_{L}^{(c)}$

$P_{R}^{(n)}=$ point of the contour line searched upslope $K_{R}^{(c)}$

if ID of the contour line containing $P_{L}^{(n)} \neq$ ID of the contour line containing $P_{R}^{(n)}$ then

\{A SADDLE is found and must be treated:\}

if a type 1 saddle is identified then

$S_{L}^{(n)}=$ point of the contour line containing $P_{L}^{(n)}$

closest to the midpoint $B_{M}$ of the associated

skeleton branch

$S_{R}^{(n)}=$ point of the contour line containing $P_{R}^{(n)}$

closest to the midpoint $B_{M}$ of the associated

skeleton branch

else if a type 2 saddle is identified then

$S_{L}^{(n)}=$ point of the contour line containing $P_{L}^{(n)}$

closest to the left endpoint $B_{L}$ of the associated

skeleton branch

$S_{R}^{(n)}=$ point of the contour line containing $P_{R}^{(n)}$

closest to the right endpoint $B_{R}$ of the

associated skeleton branch

end if

call $\operatorname{DIVIDE}\left(P_{L}^{(n)}, S_{L}^{(n)}\right)$

$P_{L}^{(n)} \leftarrow S_{R}^{(n)}$

end if

$P_{L}^{(c)} \leftarrow P_{L}^{(n)}$

$P_{R}^{(c)} \leftarrow P_{R}^{(n)}$ 
$K_{L}^{(c)}=$ point of the skeleton searched upslope $P_{L}^{(c)}$

$K_{R}^{(c)}=$ point of the skeleton searched upslope $P_{R}^{(c)}$

\section{end while}

return

[23] To summarize, the calculation is initiated by determining the first couple of current points on the contour line located immediately upward from the assigned points and calling the routine DIVIDE. Then, the routine DIVIDE is recursively called whenever a SADDLE is found. Each call terminates when skeleton points are found to be coincident, generally when a PEAK is reached.

\subsection{Flow Net Construction}

[24] The methods presented in sections 2.2 and 2.3 can be readily applied to construct a flow net from any assigned set of contour lines. Illustrative examples of flow net construction are reported in Figure 6. Figure 6a shows the solution of critical topographic structures such as ridges, saddles, and peaks represented by contour elevation data having a sufficiently fine resolution to ensure a detailed description of the terrain morphology, whereas Figure 6b shows the solution of groups of peaks and/or depressions displayed by contour elevation data having an insufficiently fine resolution to ensure a detailed description of the terrain morphology. The example provided in Figure 6a is especially intended to highlight the full automation in the analysis of complex contour elevation data allowed by skeleton construction techniques. The ordering of skeleton elements described in section 2.2 allows skeleton stems (which enrich the information provided by contour lines) and skeleton branches (which reveal topographic structures such as valleys, ridges, saddles, and peaks) to be recognized and intersected at right angles or followed, respectively, by flow lines and drainage divides. The methodology described by Gallant and Wilson [2000] can be applied to solve the case shown in Figure 6a. Regularly spaced flow lines are constructed for each pair of adjacent contour lines working form the lowest to the highest contour line. A user-specified distance controls the spacing of the flow lines and flow lines are placed at that specified distance on the first (lowest) contour line. On subsequent higher contour lines, the upper ends of the flow lines from the lower contour line are used as the start points of the next set of flow lines provided that they are neither too close nor too far apart. As suggested by Moore et al. [1988] and Moore and Grayson [1991], a new line is inserted if the distance between the existing start points is more than 1.3 times the user-specified distance, whereas a line is terminated if the start points are less than half the user-specified distance.

[25] The methodology described by Moore et al. [1988], Moore and Grayson [1991], and Gallant and Wilson [2000] is extended here in order to allow groups of peaks and/or depressions such as those considered in section 2.2 and shown in Figures $3 \mathrm{e}$ and $3 \mathrm{f}$ to be correctly processed during the construction of a flow net. Using the methods described in section 2.2, neighboring contour lines representing the same elevation are connected to obtain systems of contour lines that can be assimilated to single contour lines. Figure $3 \mathrm{e}$ shows how the closed contour lines representing the elevation $350 \mathrm{~m}$ asl (peaks $P_{1}, P_{2}$, and $P_{3}$ ) are connected to the open contour line representing the same elevation of
$350 \mathrm{~m}$ asl, whereas Figure $3 \mathrm{f}$ shows how the closed contour lines representing the elevation $345 \mathrm{~m}$ asl (depressions $D_{1}$ and $D_{2}$ ) are connected to the open contour line representing the same elevation of $345 \mathrm{~m}$ asl. The way in which the obtained systems of contour lines are traveled during the construction of a flow net is indicated by the arrows reported in Figures $3 \mathrm{e}$ and $3 \mathrm{f}$, and will be further defined in section 2.5. The flow net computed from approximately the same contour elevation data as those shown in Figures $3 \mathrm{e}$ and $3 \mathrm{f}$ is given in Figure $6 \mathrm{~b}$. The construction of this flow net is based on the methodology described above and on a recursive algorithm developed to perform flow line insertions or terminations along all the elemental systems of contour lines (representing the same elevation) that may be encountered when considering real contour elevation data.

[26] The recursive algorithm is described by referring to the flow net element $S_{1} E_{1} E_{2} S_{2}$ shown in Figure 6b, which is formed by two contour line segments $\left(S_{1} S_{2}, E_{1} E_{2}\right)$ and two flow line segments $\left(S_{1} E_{1}, S_{2} E_{2}\right)$. The algorithm starts by traveling the contour line containing the point $E_{1}$ (end of the flow line $S_{1} E_{1}$ ) in the direction indicated by the arrows reported in Figures 3e, 3f, and $6 \mathrm{~b}$ in order to find the point $E_{2}$ (end of the flow line $S_{2} E_{2}$ ). If $E_{2}$ is found, then flow lines are inserted or terminated along the contour line segment $E_{1} E_{2}$ following the rules reported above. If a connecting line is found at a given joint point (e.g., point $J$ in Figure 6b), then flow lines are inserted or terminated along the contour line segment (e.g., $E_{1} J$ in Figure $6 \mathrm{~b}$ ) before this joint point and the connecting line is traveled until the point of intersection (e.g., point $C$ in Figure 6b) with the connected contour line is found. Then, the algorithm is recursively called to process the contour line system (e.g., $C E_{2}$ in Figure $6 \mathrm{~b}$ ) lying between this point of intersection and $E_{2}$. All the recursive calls are closed when $E_{2}$ is found and flow lines are inserted or terminated along the contour line segment lying between the end of the last-considered connecting line and $E_{2}$. This recursive algorithm allows the processing of all elemental systems of contour lines, including simple contour line segments, complex systems of peaks (e.g., $P_{1}, P_{2}$, and $P_{3}$ in Figure $6 \mathrm{~b}$ ), and complex systems of depressions (e.g., $D_{1}$ and $D_{2}$ in Figure 6b).

[27] The following pseudocode gives the logic of the algorithm:

Procedure FLOWLINES $\left(E_{1}, E_{2}\right)$

Require: Contour lines, connecting lines, points $\left(E_{1}, E_{2}\right)$

Ensure: Insertions or terminations of flow lines between

$E_{1}$ and $E_{2}$

$\mathrm{CID}=\mathrm{ID}$ of the contour line containing $E_{1}$

while $E_{2}$ is not found do

Travel all elements of the contour line CID

Accumulate the distance along the contour line CID

if a connecting line is found then

Perform insertions or terminations on the contour line CID

Travel the connecting line until the connected contour line is found

$C=$ point of intersection between the connecting 
line and the connected contour line call FLOWLINES $\left(C, E_{2}\right)$

\section{end if \\ end while}

Perform insertions or terminations on the contour line CID

\section{return}

\subsection{Practical Aspects}

[28] In order to allow for easy programming and fast execution of the operations described in sections 2.2-2.4, input (contour elevation) data are arranged in such a way as to ensure that the points defining a contour line can be found in the order in which they appear to an observer moving along the contour line in the direction that keeps the upper adjacent contour line to the left (and the lower adjacent contour line to the right). This helps determine the relative location of a point with respect to other points lying on the same or on a different contour line.

[29] A practical method for ensuring a priori that the points defining the contour lines yield an accurate skeleton construction is derived from the well-defined condition reported in section 2.1 (which cannot be applied directly since it requires the skeleton itself). By observing that the skeleton stems and the skeleton branches approximately lay halfway between adjacent contour lines or between different portions of a (markedly nonstraight) contour line, respectively, the distance between a point lying on a contour line and the nearest point of the skeleton (used in the condition reported in section 2.1) may be surrogated by half the distance between the point lying on the contour line and the nearest point lying on adjacent contour lines or on other portions of the same (markedly nonstraight) contour line. Under this approximation, for each line segment defining a contour line.

[30] 1. The perpendiculars from the line segment intersecting line segments of the same and of adjacent contour lines are determined and distances are computed.

[31] 2. The perpendiculars from the line segments of the same and of adjacent contour lines intersecting the considered line segment are determined and distances are computed.

[32] 3. The spacing between the endpoints of the line segment is verified to be less than or equal to 0.125 times the minimum distance among those computed in the previous steps and, if necessary, new points are inserted inside the line segment in order to meet this requirement.

[33] Although additional work is needed to improve the method's ability to handle all possible cases in an accurate manner, the method is helpful to determine a priori a reasonable set of points that generally leads to satisfactory skeleton constructions.

[34] The program "Triangle" developed by Shewchuk [1996] is used to compute the Delaunay triangulation and the Voronoi diagram. The incircle test mentioned in section 2.1 is then applied to each Delaunay/Voronoi edge pair and skeleton line segments are grouped to form the set of skeleton stem segments (obtained from couples of Delaunay triangles having the common side with endpoints spanning on adjacent contour lines) and the set of skeleton branch segments (obtained from couples of Delaunay triangles having the common side with both endpoints lying on the same contour line). Skeleton stem segments having a shared endpoint are connected to form polylines. Each of these polylines is stored in an array using the same rule adopted for contour lines and is labeled conventionally with the mean of the upper and lower adjacent contour line elevations to define its location (Figure 3a). Skeleton branches are connected to form tree structures originating at points lying on the skeleton stems or on closed contour lines (Figures $3 \mathrm{a}$ and $3 \mathrm{~d}$ ). By relying on an ordered set of skeleton stems and skeleton branches, the method described in sections 2.3 and 2.4 can readily be applied.

\section{Method Evaluation}

[35] The methods described in section 2 are evaluated through their application to real terrains. A brief description of the terrains and of the data used to represent their morphology is reported in section 3.1. Evaluation criteria are defined in section 3.2. Section 3.3 presents the analysis carried out and the obtained results.

\subsection{Study Areas and Elevation Data}

[36] The real terrains considered in this study are referred to as the Ca' Lita area (Northern Italian Apennines, Reggio Emilia, Italy; Figures 4, 5, and 7) and the Col Rodella area (Eastern Italian Alps, Trento, Italy; Figure 8). The center of the Ca' Lita area framed in Figures 4, 5, and 7 has latitude $44^{\circ} 27^{\prime} 31^{\prime \prime} \mathrm{N}$ and longitude $10^{\circ} 38^{\prime} 38^{\prime \prime} \mathrm{E}$. In this area, the geologic substratum is constituted by clay shales of marine origin. Clay-rich soils are mostly covered by bush and grass. The topography can be described as fairly complex as a result of surface water erosion and active landsliding. Unchanneled hillside swales are not uncommon because of widespread slope deformation and movement. The elevation ranges from 323.8 to $472.0 \mathrm{~m}$ asl with an average of $393.8 \mathrm{~m}$ asl. The average terrain slope is $31.9 \%$. The center of the Col Rodella area framed in Figure 8 has latitude $46^{\circ} 29^{\prime} 39^{\prime \prime} \mathrm{N}$ and longitude $11^{\circ} 45^{\prime} 23^{\prime \prime} \mathrm{E}$. In this area, the sedimentary bedrock, primarily of limestone, crops out widely and shallow immature soils cover a steep and regular topography where only grass grows because of the altitude. Because of their coarse texture, soils are less vulnerable to surface water erosion compared to the previous case, and not at all affected by slope instability phenomena. The elevation ranges from 1836.7 to $2536.4 \mathrm{~m}$ asl with an average of $2201.4 \mathrm{~m}$ asl. The average terrain slope is $59.3 \%$.

[37] The digital topography of the $\mathrm{Ca}$ ' Lita and $\mathrm{Col}$ Rodella areas was generated from lidar surveys carried out by Helica (Italy) and Terrapoint (United States), respectively. The survey in the Ca' Lita area was carried out using an Optech ALTM (airborne lidar terrain mapping) system mounted on a helicopter. The flight altitude was about $800 \mathrm{~m}$ agl (above ground level) and the acquisition parameters were set to obtain an average data density of about 2.9 points per square meter. The positions of land surface points (determined with absolute accuracy of about $0.4 \mathrm{~m}$ and $0.15 \mathrm{~m}$, in the horizontal and vertical coordinate, respectively) were elaborated to provide $0.5-\mathrm{m}$ resolution gridded elevation data. The survey in the Col Rodella area was carried out using an ALTM system of Terrapoint's proprietary design mounted on a fixed-wing aircraft. The 

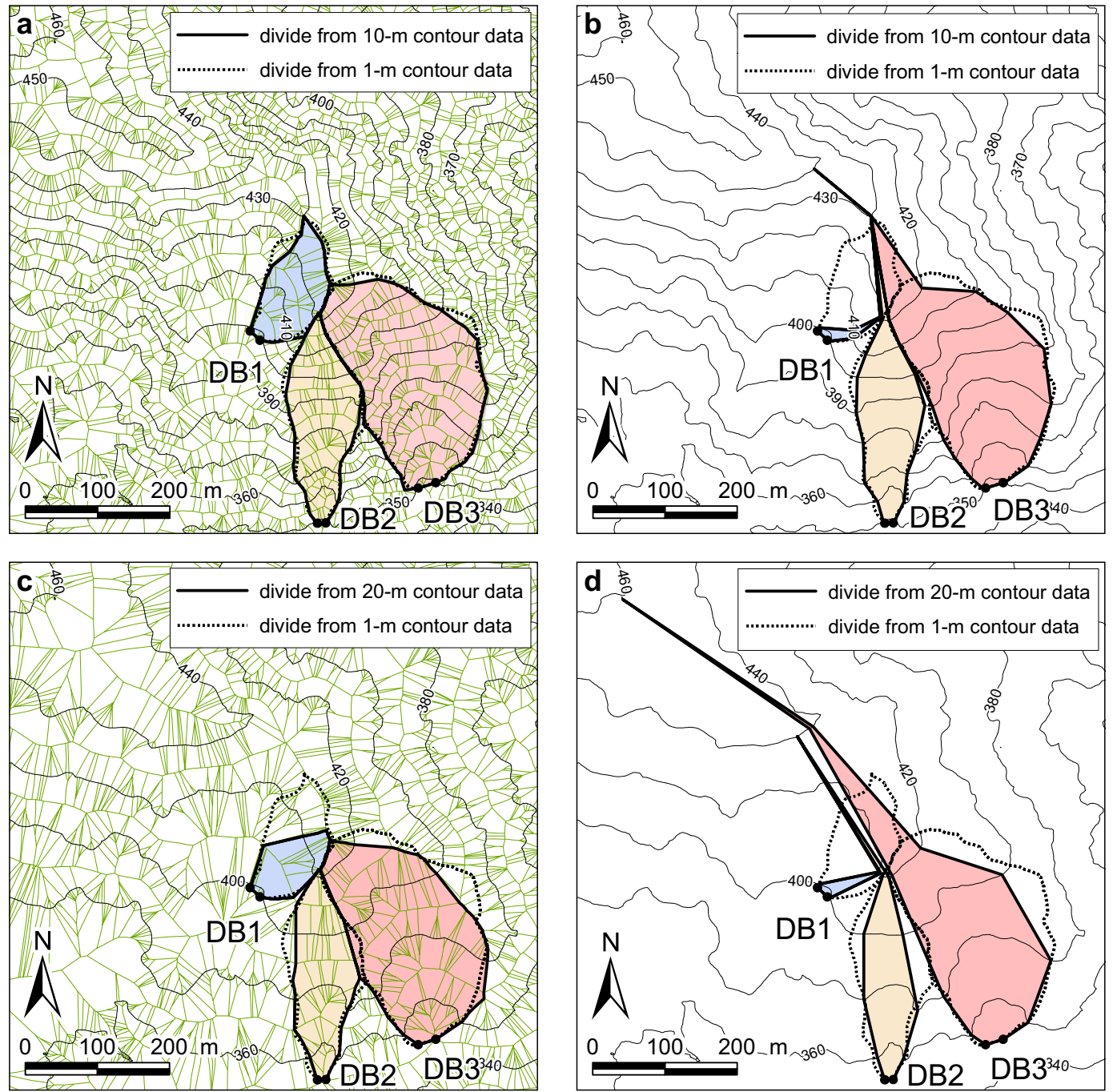

Figure 7. Delineation of drainage basins DB1, DB2, and DB3 in the Ca' Lita area $\left(44^{\circ} 27^{\prime} 31^{\prime \prime} \mathrm{N}\right.$, $10^{\circ} 38^{\prime} 38^{\prime \prime} \mathrm{E}$ ) using (a and $\mathrm{c}$ ) the proposed method and (b and d) the current state-of-the-art method with data at different contour intervals $(10 \mathrm{~m}$ in Figures $7 \mathrm{a}$ and $7 \mathrm{~b}$ and $20 \mathrm{~m}$ in Figures $7 \mathrm{c}$ and $7 \mathrm{~d})$. The reference solutions, obtained using 1-m contour data and the proposed method, are reported (in dashed lines) to facilitate comparisons. The datum is mean sea level.

flight altitude was about $1000 \mathrm{~m}$ agl and the acquisition parameters were set to obtain an average data density of about 0.5 points per square meter. The positions of land surface points (determined with absolute accuracy of about $1 \mathrm{~m}$ and $0.3 \mathrm{~m}$, in the horizontal and vertical coordinate, respectively) were elaborated to provide $1-\mathrm{m}$ resolution gridded elevation data. Gridded elevation data were resampled to $5-\mathrm{m}$ and contour elevation data with contour intervals of $1,5,10,20$, and $50 \mathrm{~m}$ were generated. The obtained data were found to offer a suitable balance between detail in the description of the terrain topography and smoothness of the derived contour lines, the latter being a desirable factor for computationally inexpensive skeleton construction.

\subsection{Evaluation Criteria}

[38] The capability of the methods described in section 2 is evaluated by verifying (visually) whether the drainage divides automatically delineated from contour elevation data of real terrains conform with the general principles that would be applied by solving the same problems manually. This evaluation criterion must be qualified by the fact that in terrain analysis the accuracy of (digital) topographic data is a critical factor affecting the extraction of primary and secondary attributes [e.g., Wilson and Gallant, 2000, p. 15]. The description of the "true topography" of a real terrain is an ideal process that can be reached only by using infinitely accurate instruments and techniques along with infinitesimal elevation model resolutions. In fact, real topographic data are always approximations of the "true topography" because of uncertainties in the use of instruments and techniques along with obvious practical constraints in elevation model resolution. Lidar (light detection and ranging) technology is able to provide much more information than can be acquired by virtually any other means, at least within economic reason [Terrapoint 

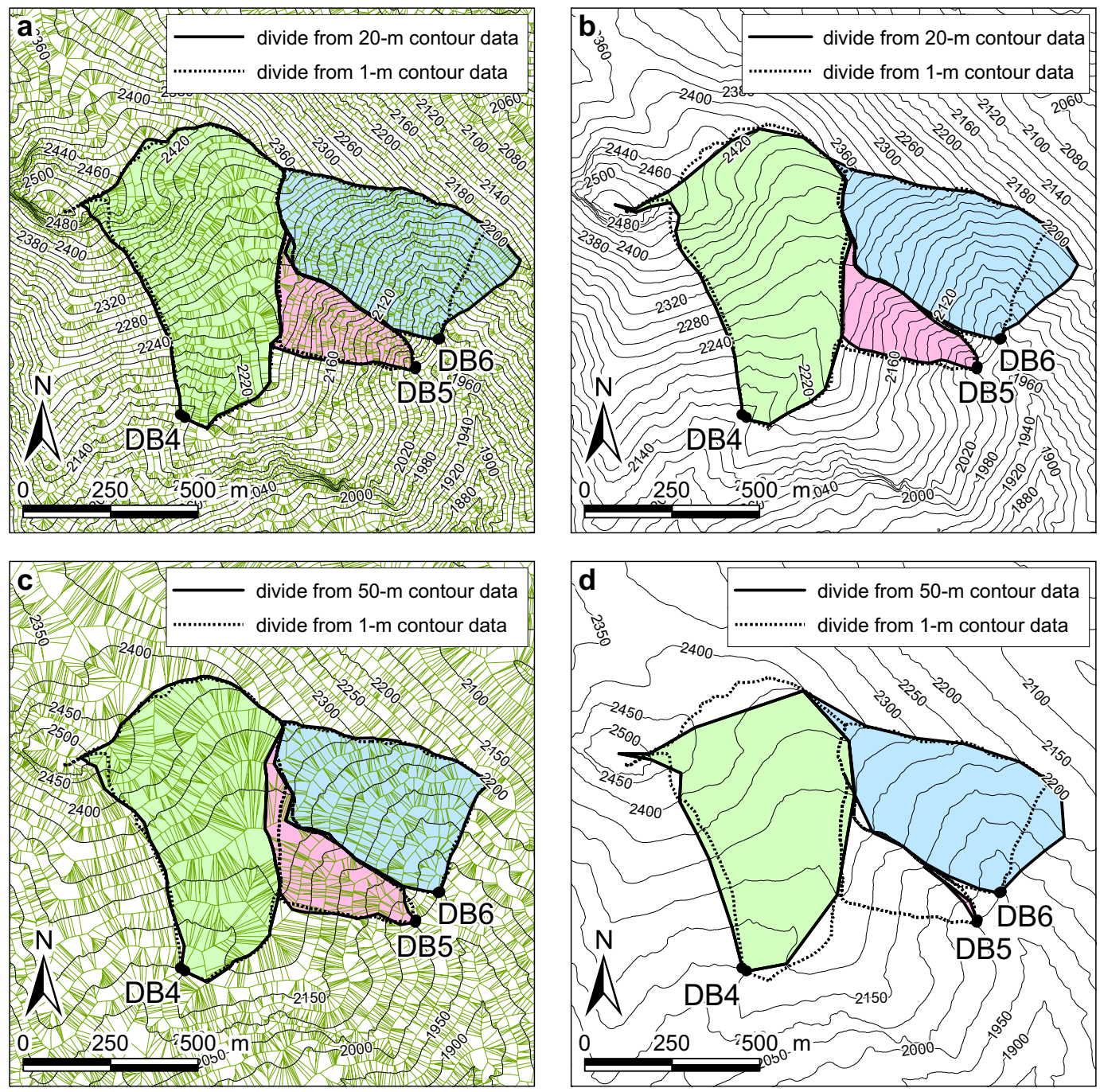

Figure 8. Delineation of drainage basins DB4, DB5, and DB6 in the Col Rodella $\left(46^{\circ} 29^{\prime} 39^{\prime \prime} \mathrm{N}\right.$, $11^{\circ} 45^{\prime} 23^{\prime \prime} \mathrm{E}$ ) area using (a and c) the proposed method and (b and d) the current state-of-the-art method with data at different contour intervals $(20 \mathrm{~m}$ in Figures $8 \mathrm{a}$ and $8 \mathrm{~b}$ and $50 \mathrm{~m}$ in Figures $8 \mathrm{c}$ and $8 \mathrm{~d})$. The reference solutions, obtained using 1-m contour data and the proposed method, are reported (in dashed lines) to facilitate comparisons. The datum is mean sea level.

USA Inc., 2005]. To investigate in some way the relative role of data and method accuracy in the delineation of drainage basins, contour elevation data with different contour intervals are derived in this study from lidar surveys as mentioned in section 3.1, and results obtained from the application of the proposed method are compared with those obtained by applying a "state-of-the-art method" incorporating the relevant concepts and techniques currently in use [e.g., Moore et al., 1988; Dawes and Short, 1994; Maunder, 1999; Menduni et al., 2002]. Both the current state-of-the-art and proposed methods are essentially based on the same general principle (i.e., minimum distance searching upslope mentioned in section 1), the essential difference between the two being that the second exploits the skeleton of contour lines as a means for reconstructing a plausible morphology of the terrain lying within adjacent contour lines or within a closed contour line whereas the first does not (section 2.3, Figures 4 and 5).
[39] The improvement in the delineation of drainage basins offered by the proposed method with respect to the current state-of-the-art method can also be evaluated numerically by considering the areas $A_{1}, A_{2}$, and $A_{3}$ shown in Figure 9. In Figure 9, two drainage basin determinations briefly denoted as "DBD1" and "DBD2" are sketched. DBD1 and DBD2 are obtained by considering the same draining line segment connecting two assigned points $\left(P_{L}\right.$ and $P_{R}$ ), but by different methods. Area $A_{1}$ is the portion of DBD1 that does not belong to DBD2. Area $A_{2}$ belongs to both DBD1 and DBD2 (intersection). Area $A_{3}$ is the portion of DBD2 that does not belong to DBD1. Areas of DBD1 and DBD2 are $A_{1}+A_{2}$ and $A_{2}+A_{3}$, respectively, the overlapping area is $A_{2}$, and the nonoverlapping areas are $A_{1}$ and $A_{3}$. Areas $A_{1}, A_{2}$, and $A_{3}$ allow one to define two types of errors, both relative to the area of DBD1 $\left(A_{1}+A_{2}\right)$, which is taken as reference. The type 1 relative error $\mid\left(A_{2}+A_{3}\right)-$ 


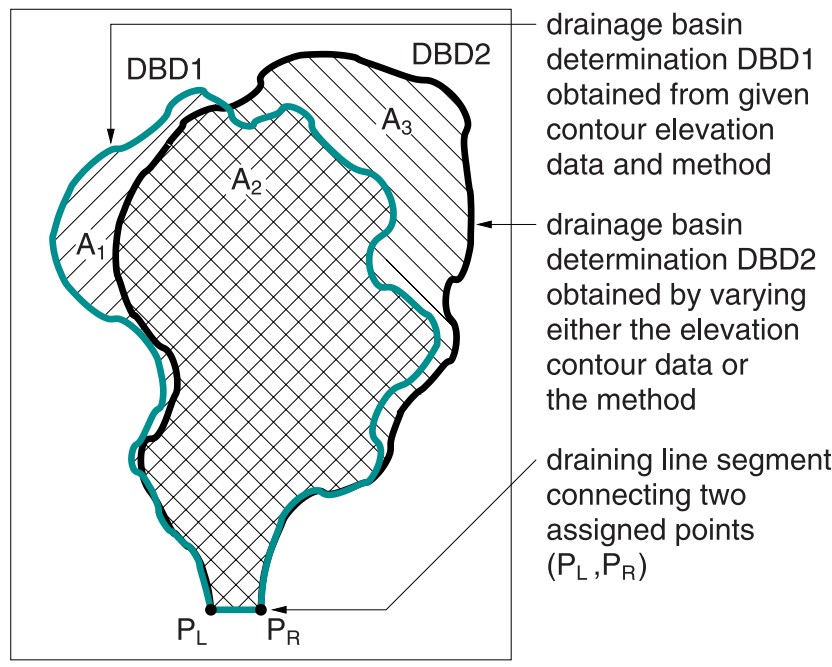

Figure 9. Comparison between drainage basin determinations "1" and "2" (DBD1 and DBD2, respectively) obtained by applying two different methods.

$\left(A_{1}+A_{2}\right) \mid /\left(A_{1}+A_{2}\right)$ accounts for the absolute difference between the areas of DBD1 and DBD2 and is given by

$$
E_{1}=\frac{\left|A_{1}-A_{3}\right|}{A_{1}+A_{2}} .
$$

[40] The type 2 relative error accounts for the total nonoverlapping area $\left(A_{1}+A_{3}\right)$ and is given by

$$
E_{2}=\frac{A_{1}+A_{3}}{A_{1}+A_{2}} .
$$

[41] One can note that error $E_{1}$ is just an indicator of the absolute difference between the drainage areas of DBD1 and DBD2, independently on the location of these two basin determinations, whereas error $E_{2}$ also accounts for the nonoverlapping between the two basin determinations. Since $A_{1}+A_{3} \geq\left|A_{1}-A_{3}\right|$, it follows that $E_{2} \geq E_{1}$. If both the areas and locations of DBD1 and DBD2 differ, then both errors $E_{1}$ and $E_{2}$ are greater than zero. If DBD1 and DBD2 have the same drainage area $\left(A_{1}+A_{2}=A_{2}+A_{3}\right)$, but different locations, then error $E_{1}$ is equal to zero $\left(A_{3}=A_{1}\right)$ and error $E_{2}$ is greater than zero, since $A_{1}>0$ and $A_{3}>0$. If DBD1 and DBD2 overlap perfectly, then errors $E_{1}$ and $E_{2}$ are both equal to zero, since $A_{1}=0$ and $A_{3}=0$.

\subsection{Analysis and Results}

[42] Among all the generated sets of contour lines mentioned in section 3.1, that having contour interval of $1 \mathrm{~m}$ is expected to provide the best approximation of the true terrain topography. Accordingly, the solution obtained by using 1-m contour data and the proposed method is taken as a point of reference for evaluating the other solutions obtained by varying either the contour interval or the method. Six test case drainage basins are considered. Three of them, denoted as DB1, DB2, and DB3, are located in the Ca' Lita area (Figures 4, 5, and 7), whereas the other three, denoted as DB4, DB5, and DB6, are located in the Col Rodella area (Figure 8). The drainage areas of DB1, DB2, DB3, DB4, DB5, and DB6, are 11,982.6, 19,175.6, 43,718.7, 298,688.1, 68,417.2, and
179,286.6 $\mathrm{m}^{2}$, respectively. These six drainage basins provide a sufficiently large set of test cases for the purpose of the present study, which is to illustrate the salient features of the proposed method for different geomorphological settings. A first test is carried out by delineating the drainage basins DB1, DB2, and DB3 in the Ca' Lita area using $1-\mathrm{m}$ contour data (Figures 4 and 5). To highlight the capability of the proposed method, the delineation of drainage basins DB1, DB2, and DB3 in the Ca' Lita area is carried out by using contour elevation data with contour intervals greater than $1 \mathrm{~m}$. Results obtained by using $10-\mathrm{m}$ and $20-\mathrm{m}$ contour data are shown in Figure 7. The delineation of the drainage basins DB4, DB5, and DB6 in the Col Rodella area by using $20-\mathrm{m}$ and $50-\mathrm{m}$ contour data are shown in Figure 8. The reference solutions, obtained by using 1-m contour data and the proposed method, are reported (in dashed lines) in Figures 7 and 8 to facilitate comparisons. Errors $E_{1}$ and $E_{2}$ are evaluated for the six test case drainage basins by considering the application of the current state-of-the-art and proposed methods to contour elevation data with contour interval of 1, 5, 10, 20, and $50 \mathrm{~m}$. Results are shown in Figure 10.

[43] The computational burden imposed by the proposed method can be evaluated by considering separately the two contributions to total central processing unit (CPU) time required for (1) skeleton extraction from contour lines and (2) drainage basin delineation or flow net construction. The CPU time required to extract the skeleton from contour lines rapidly decreases as contour interval increases. Using an Intel Core 2 Duo 6700 processor, the CPU time required to extract the skeleton from contour lines representing the area framed in Figures 4 and 7 decreases form $2.43 \mathrm{~h}$ to $5.0 \mathrm{~min}$ as the contour interval increases from 1 (Figure 4) to $10 \mathrm{~m}$ (Figure $7 \mathrm{a}$ ). The $\mathrm{CPU}$ time required to delineate a simple drainage basin is of the order of a few seconds for both proposed and current state-of-the-art methods. However, if 1-m contour data are used and specific checks are made in order to attempt to solve complex topographic structures (such as those shown in Figure 4) using the current state-of-the-art method, the CPU time may go beyond $1 \mathrm{~h}$ (and accurate results are not always achieved). In this context, the computational burden imposed by skeleton extraction from contour lines appears quite bearable. In scientific studies, CPU times of the order of a few hours appear acceptable in exchange for (accurate) solutions from fine resolution elevation data. In technical applications, CPU times of the order of a few minutes appear acceptable in exchange for accurate solutions from coarse resolution elevation data.

\section{Discussion and Conclusions}

[44] The use of the skeleton of a set of contour lines offers important improvements in the delineation of drainage basins and construction of flow nets over solutions provided by the current state-of-the-art method. First, divide elements between each pair of adjacent contour lines can normally be determined in such a way as to ensure the perpendicularity to both the upper and lower contour lines (bottom inset in Figures 4 and 5 and Figure 6). This capability allows for a higher accuracy in the delineation of drainage basins and ensures the uniqueness of the solution with respect to the use of an upslope or downslope searching algorithm. 

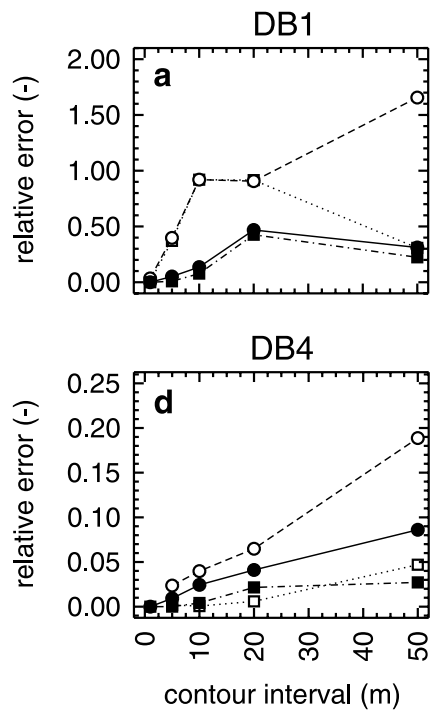
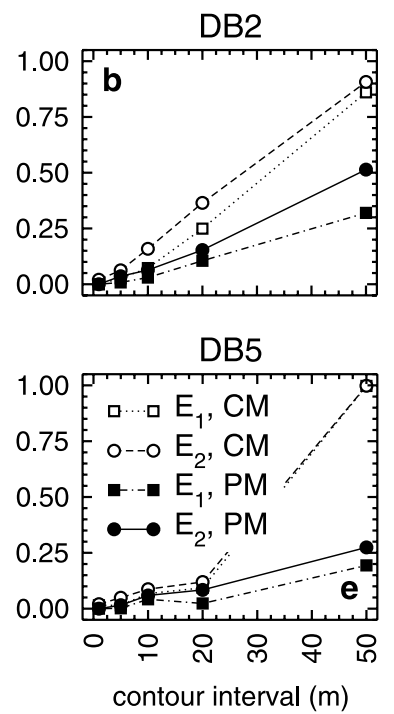
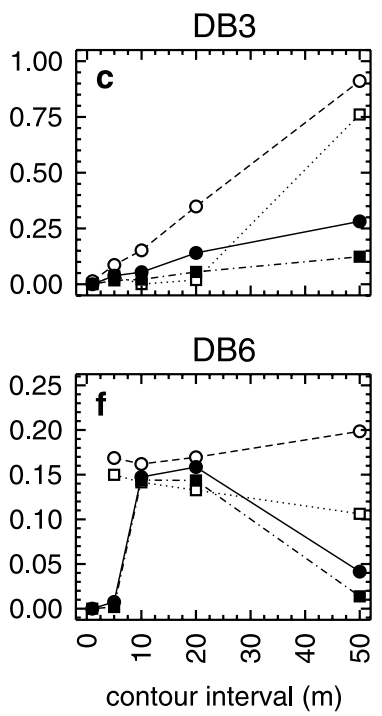

Figure 10. Type 1 and type 2 relative errors for the current state-of-the-art method (denoted here as $\mathrm{CM}$ ) and the proposed method (PM) using contour elevation data at different contour intervals. Drainage basins (a) DB1, (b) DB2, and (c) DB3 in the Ca' Lita area and drainage basins (d) DB4, (e) DB5, and (f) DB6 in the Col Rodella area are considered.

Secondly, critical topographic structures such as ridges, saddles, and peaks are solved more accurately by exploiting the skeleton structure (top insets in Figures 4 and 5 and Figure 6). The skeleton structure allows morphological features that may be recognized by human discernment on the basis of a set of contour lines to be explicitly revealed and incorporated in a computer program. Thirdly, full automation in the analysis of contour elevation data is achieved by relying on the skeleton structure, since recursive algorithms of the kind presented in section 2.3 are not always successful over a set of contour lines alone. The degree of automation allowed by the proposed method was tested on some real cases and it is highlighted in the examples of flow net construction shown in Figure 6.

[45] Drainage divides obtained from the proposed method conform with the general principles that would be applied by an expert hydrologist in solving the same problems manually. Results obtained using 1-m contour data are totally satisfactory (Figure 4) and provide some improvements with respect to the results obtained by applying the current state-of-the-art method (Figure 5). The improvements offered by the new method are even more significant for the contour elevation data with contour intervals of 5,10 , 20 , and $50 \mathrm{~m}$ (Figures 7, 8, and 10). One can note that the gain in accuracy is not only due to the skeleton stems as a sort of contour data enrichment, but also to the methodological advance allowed by a profitable use of the entire skeleton structure (stems and branches). In fact, the solution provided by the proposed method using $20-\mathrm{m}$ contour data (Figure 7c) is more accurate than the solution provided by the current state-of-the-art method using 10-m contour data (Figure 7b). The results obtained for the drainage basins DB4, DB5, and DB6 in the Col Rodella area confirm these conclusions (Figure 8).

[46] The comparison between drainage basin determinations obtained by varying the contour interval of contour elevation data reveals that relative errors with respect to the reference solution (obtained using $1-\mathrm{m}$ contour data and the proposed method) generally increase as the contour interval increases (Figures 7, 8, and 10). However, in the cases of the drainage basins DB1 and DB6 (Figures 10a and 10f, respectively), the solution provided by the proposed method in $50-\mathrm{m}$ contour data is closer to the reference solution than the solution obtained in $20-\mathrm{m}$ contour data. This fact indicates that the role played by the detail in the topographic representation of a terrain is not always susceptible to quantification. More importantly, relative errors in the delineation of drainage basins are connected to terrain complexity as indicated, for instance, by the degree of ramification (branching) of the skeleton. In this perspective, the greater complexity of the Ca' Lita area (Figure 7) with respect to the Col Rodella area (Figure 8) explains, at least in part, the larger relative errors obtained for drainage basins DB1, DB2, and DB3 (Figures 10a, 10b, and 10c, respectively) with respect to those obtained for drainage basins DB4, DB5, and DB6 (Figures 10d, 10e, and 10f, respectively). As a general indication, using the proposed method and contour elevation data with contour intervals of 5-50 m, relative errors in the delineation of drainage basins are estimated to be normally less $20 \%$ and rarely greater than 50\% (Figure 10).

[47] The conclusions of the present study are summarized as follows: (1) the proposed method allows fully automated delineations of drainage basins and constructions of flow nets from contour elevation data, even when critical topographic structures such as ridges, saddles, and peaks are present; (2) for any given set of contour lines, the proposed method provides more accurate solutions than previously proposed methods, the gain in accuracy normally increasing as the contour interval increases; and (3) skeleton construction techniques allow the morphological information implicitly present in contour elevation data to be explicitly revealed and correctly processed by a computer program, and are a useful means for improving the accuracy with which physiographic features of drainage basins are deter- 
mined. The proposed methods can be used to advance the delineation of drainage basins and the construction of flow nets (as outlined in this paper), and to test the reliability of algorithms in the analysis of more efficient and straightforward, gridded or triangulated, elevation data (as will be shown in a future companion paper).

\section{Availability}

[48] The FORTRAN 90 codes that implement the methods presented in this paper are freely available from the authors under the GNU General Public License agreement.

[49] Acknowledgments. The program "Triangle" by Jonathan R. Shewchuk (University of California, Berkeley, United States) was used in this study for the computation of Delaunay triangulations and Voronoi diagrams. The authors thank Maurizio Cingi (Enìa, Italy) for advice during the development of FORTRAN 90 codes as well as Claudio Paniconi and John F. V. Riva (INRS-ETE, Université du Québec, Canada) for helpful comments on an early version of the manuscript. The authors are grateful to John Gallant (CSIRO, Australia) and two anonymous reviewers for their insightful and detailed comments.

\section{References}

Amenta, N., M. Bern, and D. Eppstein (1998), The crust and the $\beta$-skeleton: Combinatorial curve reconstruction, Graphical Models Image Process., $60(2), 125-135$.

Blum, H. (1967), A transformation for extracting new descriptors of shape, in Models for the Perception of Speech and Visual Form, edited by W. W. Dunn, pp. 153-171, MIT Press, Cambridge, Mass.

Dakowicz, M., and C. Gold (2003), Extracting meaningful slopes from terrain contours, Int. J. Comput. Geom. Appl., 13(4), 339-357.

Dawes, W. R., and D. Short (1994), The significance of topology for modeling the surface hydrology of fluvial landscapes, Water Resour. Res., 30(4), 1045-1055.

Delaunay, B. (1934), Sur la sphère vide, Izv. Akad. Nauk SSSR, 7, 793-800.

Fabbri, R., L. F. Estrozi, and L. da F. Costa (2002), On Voronoi diagrams and medial axes, J. Math. Imaging Vision, 17(1), 27-40.

Gallant, J. C., and J. P. Wilson (2000), Primary topographic attributes, in Terrain Analysis: Principles and Applications, edited by J. P. Wilson and J. C. Gallant, pp. 51-85, John Wiley, New York.

Gold, C. M. (1999), Crust and anti-crust: A one-step boundary and skeleton extraction algorithm, paper presented at Conference on Computational Geometry, Assoc. for Comput. Mach., Miami, Fla.
Gold, C. M., and J. Snoeyink (2001), A one-step crust and skeleton extraction algorithm, Algorithmica, 30(2), 144-163.

Kirkpatrick, D. G., and J. D. Radke (1988), A framework for computational morphology, in Computational Geometry, edited by G. Toussaint, pp. 217-248, North-Holland, Amsterdam.

Leopold, L. B., M. G. Wolman, and J. P. Miller (1964), Fluvial Processes in Geomorphology, W. H. Freeman, San Francisco, Calif.

Maunder, C. J. (1999), An automated method for constructing contourbased digital elevation models, Water Resour. Res., 35(12), 3931-3940.

Maxwell, J. C. (1870), On hills and dales, London Edinburgh Dublin Philos. Mag. J. Sci., 4/40(269), 421-427.

Menduni, G., and V. Riboni (2000), A physically based catchment partitioning method for hydrological analysis, Hydrol. Processes, 11-12(2), $1943-1962$.

Menduni, G., A. Pagani, M. C. Rulli, and R. Rosso (2002), A nonconventional watershed partitioning method for semi-distributed hydrological modelling: The package ALADHYN, Hydrol. Processes, 16(2), $277-291$.

Moore, I. D., and R. B. Grayson (1991), Terrain-based catchment partitioning and runoff prediction using vector elevation data, Water Resour. Res., 27(6), 1177-1191.

Moore, I. D., E. M. O’Loughlin, and G. J. Burch (1988), A contour-based topographic model for hydrological and ecological applications, Earth Surf. Processes Landforms, 13(4), 305-320.

O'Loughlin, E. M. (1986), Prediction of surface saturation zones in natural catchments by topographic analysis, Water Resour. Res., 22(5), 794-804.

Onstad, C. A., and D. L. Brakensiek (1968), Watershed simulation by stream path analogy, Water Resour. Res., 4(5), 965-971.

Shewchuk, J. R. (1996), Triangle: Engineering a 2D quality mesh generator and Delaunay triangulator, in Applied Computational Geometry: Towards Geometric Engineering, Lect. Notes Comput. Sci., vol. 1148, edited by M. C. Lin and D. Manocha, pp. 203-222, Springer, Berlin.

Terrapoint USA Inc. (2005), A white paper on LiDAR mapping, 10 pp., Terrapoint USA Inc., The Woodlands, Tex.

Thibault, D., and C. M. Gold (2000), Terrain reconstruction from contours by skeleton construction, GeoInformatica, 4(4), 349-373.

Voronoi, G. (1907), Nouvelles applications des paramètres continus à la théorie des formes quadratiques, J. Reine Angew. Math., 133, 97-178.

Wilson, J. P., and J. C. Gallant (2000), Digital terrain analysis, in Terrain Analysis: Principles and Applications, edited by J. P. Wilson and J. C. Gallant, pp. 1-27, John Wiley, New York.

G. Moretti and S. Orlandini, Dipartimento di Ingegneria Meccanica e Civile, Università degli Studi di Modena e Reggio Emilia, Via Vignolese 905, I-41100 Modena, Italy. (giovanni.moretti@unimore.it; stefano.orlandini@ unimore.it) 